\title{
On the uniqueness of the Einstein-Straus model
}

\author{
$\operatorname{Marc~Mars~}^{1}$ \\ Albert Einstein Institut, Am Mühlenberg 1, D-14476 Golm, Germany
}

Received 25 June 2001

Published 15 August 2001

Online at stacks.iop.org/CQG/18/3645

\begin{abstract}
We show that the Einstein-Straus model does not give a robust answer to the problem of the influence of the cosmic expansion on the local physics. This is done by finding the most general static region embeddable in a FriedmannLemaitre-Robertson-Walker expanding cosmology and showing that the model must be 'almost spherically symmetric'. More precisely, we show that the boundary of the static region must be a 2 -sphere at each instant of cosmic time. The motion of this 2-sphere in spacetime is as follows: its 'would-be' centre (if there were no static region) moves along a path whose projection on any 3space of constant cosmic time is a geodesic with respect to the induced 3-metric. The velocity of this geodesic is determined from the matching. In particular, this centre must be at rest with respect to the cosmological flow (thus giving a spherically symmetric model) when any of the standard energy-momentum tensors inside the static region is imposed.
\end{abstract}

PACS numbers: 0420J, 0420C, 0440N, 9880

\section{Introduction}

The problem of the influence of the cosmic expansion on our local physics has received repeated attention since long ago. Although this effect is generally believed to be either non-existent or very small (so that it can be ignored for all practical purposes), this issue does not have yet a satisfactory answer within general relativity. Indeed, 'no effect on the local physics' has been taken to mean that the geometry around us is static and it is a priori unclear whether this is compatible with a Friedmann-Lemaitre-Robertson-Walter (FLRW) standard cosmology at larger scales. The first attempt to model this situation is due to McVittie [1], who presented a metric claimed to represent a point particle in a FriedmannLemaître background. His conclusion, later extended and improved by Järnefeld [2], was that the influence of the cosmic expansion was very small indeed. The interpretation of McVittie's metric as describing a point particle was disputed later on by Sussman [3] and this question is still being investigated (see Nolan [4]). Nevertheless, the generally accepted solution to

\footnotetext{
1 Also at Laboratori of Fisica Matemàtica, IEC, Barcelona, Spain.
} 
the influence of the expansion on the local physics was given by Einstein and Straus, who showed that the Schwarzschild metric can be matched to an expanding FLRW dust model. This is the so-called Einstein-Straus (ES) [5], also known as Swiss-cheese, model. However, this approach is known to have several important limitations. First, the geometry of the cavity is assumed to be exactly spherically symmetric. This is a very restrictive and little justified assumption. Studying whether this can be relaxed or not, and to what extent, will be one of our main aims in this paper. Secondly, the central mass in the static region turns out to be directly related to the radius of the cavity and also to the background density of the cosmological model. As discussed by Bonnor [6], this relationship severely restricts the kind of objects to which the ES model can be applied. In particular, it is not suitable for solar system scales [6]. Thirdly, the model is known to be unstable under radial perturbations (see Krasiński [7]).

These drawbacks of the ES model have triggered new activity in this topic. In a very interesting paper, Bonnor [8] studied the effect of the cosmic expansion on the size of the hydrogen atom. This was done by analysing the Maxwell-field equations in a FriedmannLemaitre cosmology. The conclusion was that the change in size of the hydrogen atom is very small compared with the size of the atom itself (see also [9] for additional comments on this paper). In another paper, Bonnor [10] relaxes the constraint between the central mass and the density of the background by considering the matching between a Schwarzschild metric and a Lemaitre-Tolman-Bondi model (which is an inhomogeneous, spherically symmetric generalization of the dust FLRW cosmology). It turns out that no restriction on the size of the cavity or on the background density exists in this situation. Furthermore, although the model is again unstable, the instability time scale is very long, even when compared with cosmic time scales.

All of these models, however, assume spherical symmetry from the outset. So, it is necessary to analyse what happens when this condition is dropped. The first attempt to address this issue is due to Senovilla and Vera [11], who found a surprising result, namely that the cylindrically symmetric analogue to the ES model is impossible, even when the matter content of the static region is left arbitrary. In other words, a static and cylindrically symmetric spacetime cannot be matched to a FLRW model across a cylindrically symmetric boundary. This indicated that, possibly, the condition of spherical symmetry was crucial for the existence of the ES model. To answer this question in a conclusive way, the existence problem of static regions (with no further symmetry) embedded in a FLRW background needs to be analysed. This is the aim of this paper. A first step in this direction was made in [12] where the most general static and axially symmetric, orthogonally transitive (see, e.g., [13] for a definition), spacetime satisfying an additional constraint between the cosmic and the static times on the boundary, was studied. This condition on the times was general enough to include both the ES model and the cylindrically symmetric model (if this existed). In particular, this condition did not imply that the rate of cosmic time and the rate of static time are the same on the boundary (such a condition is violated for the ES model, see [14]). The result obtained in [12] was that non-spherically symmetric models $d o$ exist, but they must have a very special 'almost spherically symmetric' geometry. More precisely, the boundary of the static region has to be a 2-sphere in the FLRW background whose centre moves along a geodesic in space (i.e. the projection on any hypersurface of constant cosmic time of the worldline of this centre is a geodesic of the induced metric). The radius of this sphere is arbitrary but the speed of its centre is uniquely determined by the radius function and the initial velocity. Furthermore, the static metric is completely and uniquely determined by the radius function. Its Petrov type is $\mathrm{D}$ and its energy-momentum tensor has the same algebraic type as for a spherically symmetric spacetime. Moreover, imposing any of the usual matter models inside the static region forces the whole model to be spherically symmetric. This result indicated rather strongly that nonspherical models, although possible, are very special and unphysical. However, the fact that 
the static region was assumed to satisfy a priori certain conditions (namely, axial symmetry and the condition on the times) prevented those results from being conclusive (the restrictions were mathematically convenient rather than physically motivated). Thus, it would be of interest to relax those conditions and study static regions in a FLRW background in full generality, with no assumptions on the geometry of the static region or on the shape of the boundary. This is the problem we solve in this paper.

By exploiting carefully the matching conditions between two spacetimes and their geometrical consequences, we prove that the axially symmetric model with coincident times on the boundary is the most general model describing a static cavity in an expanding standard cosmological model. We make this statement precise in theorem 1 below, which summarizes the results of this paper. The conclusion, therefore, is that the spherically symmetric ES model is exceptional and that the assumption of spherical symmetry is crucial for its existence. Thus, the ES model cannot give a robust answer to the question of the influence of the cosmic expansion on the local physics. Consequently, other methods should be used to clarify this question. It is likely that a correct understanding of this issue can only be achieved after a proper solution of the so-called averaging problem in general relativity is found. Indeed, the averaging problem lies at the very heart of the question of how local metrics around astrophysical objects organize themselves to give rise to a homogeneous and isotropic spacetime at large scales. Although there have been several attempts to address this difficult problem (see [15] and references therein), none of them gives a satisfactory answer as yet and much more work is necessary.

The paper is organized as follows. In section 2 we give a brief summary of the matching conditions across hypersurfaces of arbitrary causal character, which we will need later. We also recall very briefly a $2+1$ decomposition of the matching conditions put forward in [12]. In section 3 , we concentrate on the restrictions imposed by the matching conditions on the FLRW region of the spacetime. In section 4 we determine completely the Riemann tensor of the static region. This is achieved without introducing any specific coordinate system so that the expressions have a geometrically transparent meaning. Finally, section 5 is devoted to stating and proving theorem 1 , which is the main result of this paper. This theorem gives, in particular, the explicit form of the static metric in some neighbourhood of the matching hypersurface. In the appendix we obtain the explicit form of the metric in warped product spacetimes $\mathbb{R} \times \mathcal{K}$ in certain 'moving' coordinate systems. This result is used in the main text and might also be of independent interest.

\section{A brief summary of matching conditions through hypersurfaces of arbitrary causal character}

Gluing two spacetimes ${ }^{2}$ consists in taking a pair of $C^{2}$ spacetimes $\left(V^{ \pm}, g^{ \pm}\right)$with $C^{3}$ boundaries $\Omega^{ \pm}$and identifying the points and the tangent spaces on these boundaries. Thus, a diffeomorphism between $\Omega^{+}$and $\Omega^{-}$must exist satisfying the matching conditions, also called Darmois [16] junction conditions. A formalism valid for hypersurfaces of arbitrary causal character (even a changing one) was given in [17]. Since we shall use it extensively, let us summarize it briefly.

As $\Omega^{ \pm}$are diffeomorphic to each other, there exists an abstract three-dimensional $C^{3}$ manifold $\Omega$ and two $C^{3}$ embeddings

$$
\begin{array}{ll}
\Phi_{+}: & \Omega \longrightarrow V^{+}, \\
\Phi_{-}: & \Omega \longrightarrow V^{-},
\end{array}
$$

2 A $C^{n}$ spacetime is a paracompact, Haussdorf, connected $C^{n+1}$ manifold with a Lorentzian metric of class $C^{n}$. Our convention for the signature is $\{-1,1,1,1\}$. 
which satisfy $\Phi_{+}(\Omega)=\Omega^{+}$and $\Phi_{-}(\Omega)=\Omega^{-}$. The matching conditions split into two sets. First, the isometry of $\Omega^{ \pm}$with their induced metrics, i.e.

$$
\Phi_{+}^{\star}\left(g^{+}\right)=\Phi_{-}^{\star}\left(g^{-}\right)
$$

where $\Phi^{\star}$ denotes the pull-back of $\Phi$. The second set requires choosing two $C^{2}$ vector fields $\vec{l}_{ \pm}$on $\Omega^{ \pm}$(called riggings) which are transverse everywhere to the boundaries, with different relative orientation (i.e. either $\vec{l}_{+}$points outside $V^{+}$and $\vec{l}_{-}$points inside $V^{-}$or vice versa) and satisfying

$$
\Phi_{+}^{\star}\left(l_{+}\right)=\Phi_{-}^{\star}\left(l_{-}\right), \quad \Phi_{+}^{\star}\left(l_{+}\left(\vec{l}_{+}\right)\right)=\Phi_{-}^{\star}\left(l_{-}\left(\vec{l}_{-}\right)\right),
$$

where $l_{ \pm}=g^{ \pm}\left(\vec{l}_{ \pm}, \cdot\right)$. The existence of the riggings $\vec{l}_{ \pm}$is easy to establish when the boundaries $\Omega^{ \pm}$are everywhere spacelike or timelike (they can be taken, for instance, as the unit normal vectors, with the appropriate orientation), but they may not exist when the boundaries have null points. Once this choice has been made, the second set of matching conditions demands the equality of the pull-back of the covariant derivative of the rigging 1-forms, i.e.

$$
\Phi_{+}^{\star}\left(\nabla^{+} l_{+}\right)=\Phi_{-}^{\star}\left(\nabla^{-} l_{-}\right)
$$

where $\nabla^{ \pm}$is the Levi-Civita covariant derivative of $\left(V^{ \pm}, g^{ \pm}\right)$. It can be proven [17] that these conditions are satisfied for one pair of riggings if and only if they are satisfied by any choice of riggings. Furthermore, the covariant derivatives in (3) can be substituted by the symmetrized covariant derivatives without loss of generality.

When the matching conditions are fulfilled, there exists a $C^{0}$ spacetime $(V, g)$ with the following properties. The manifold $V$ is the union of $V^{+}$and $V^{-}$with the points in the boundaries identified (we shall abuse the notation and call $\Omega$ the image of the two boundaries $\Omega^{ \pm}$in $V$ ). The metric $g$ is piecewise $C^{2}$ and the Riemann tensor has finite one-side limits on $\Omega$. Moreover [17], there exists a symmetric tensor $B_{\alpha \beta}$, defined on $\Omega$, such that the one-side limits of the Riemann tensor on $\Omega$ satisfy

$$
R_{\alpha \beta \lambda \mu}^{+}=R_{\alpha \beta \lambda \mu}^{-}+n_{\alpha} n_{\lambda} B_{\beta \mu}-n_{\beta} n_{\lambda} B_{\alpha \mu}-n_{\alpha} n_{\mu} B_{\beta \lambda}+n_{\beta} n_{\mu} B_{\alpha \lambda}
$$

in any $C^{1}$ coordinate system covering $\Omega$ (or part thereof). In this expression, $R_{\alpha \beta \lambda \mu}^{ \pm}$denotes the Riemann tensors of $\left(V^{ \pm}, g^{ \pm}\right)$evaluated on $\Omega$ and $n_{\alpha}$ is a normal 1-form to the hypersurface $\Omega$. The tensor $B_{\alpha \beta}$ is defined up the transformation

$$
B_{\alpha \beta} \rightarrow B_{\alpha \beta}+X_{\alpha} n_{\beta}+X_{\beta} n_{\alpha} .
$$

In general, a matching between two spacetimes consists in finding two embeddings $\Phi_{ \pm}$ satisfying all the conditions above. For problems with a high degree of symmetry, imposing and solving the matching conditions is, generally, a not-too-difficult problem. When little or no symmetry is present, the matching conditions become a set of coupled partial differential equations (PDEs) which is, in general, difficult to solve. A method that sometimes can be useful to tackle such problems consists in splitting the matching conditions in a $2+1$ fashion. The idea is to foliate $\left(\Omega, \Phi_{-}^{\star}\left(g^{-}\right)\right)$with a set of spacelike 2 -surfaces $S_{\tau}$, where $\tau \in \mathbb{R}$. Then, the matching conditions impose restrictions on $S_{\tau}$, called constraint matching conditions, which are sometimes easier to study than the full set of conditions on $\Omega$. Very briefly, they consist in the following (see section II of [12] for details). Let $i_{\tau}: S_{\tau} \longrightarrow \Omega$ be the inclusion map of $S_{\tau}$ into $\Omega$. The compositions $\Phi_{\tau, \pm} \equiv \Phi_{ \pm} \circ i_{\tau}$ define embeddings of $S_{\tau}$ into $\left(V^{ \pm}, g^{ \pm}\right)$. By construction, the images $S_{\tau}^{ \pm} \equiv \Phi_{\tau, \pm}\left(S_{\tau}\right)$ are spacelike 2-surfaces lying on the boundary of $V^{ \pm}$. Let us denote by $\left(N S_{\tau}^{ \pm}, S_{\tau}^{ \pm}, \pi_{ \pm}\right)$the normal bundle to the surface $S_{\tau}^{ \pm}$, i.e. the bundle with base $S_{\tau}^{ \pm}$such that the fibre at any point $x \in S_{\tau}^{ \pm}$is the space of normal 1-forms of $S_{\tau}^{ \pm}$ 
at $x$. We denote this fibre, which is a two-dimensional Lorentzian vector space, by $N_{x} S_{\tau}^{ \pm}$. The constraint matching conditions consist also of two parts. Firstly, the induced metrics on $S_{\tau}$ must coincide, i.e.

$$
\Phi_{\tau,+}^{\star}\left(g^{+}\right)=\Phi_{\tau,-}^{\star}\left(g^{-}\right)
$$

Secondly, let $x \in S_{\tau}$ be an arbitrary point and consider the fibres of $N S_{\tau}^{ \pm}$at the points $\Phi_{\tau, \pm}(x)$, which we still denote by $N_{x} S_{\tau}^{ \pm}$. There must exist a linear and isometric map

$$
f_{\tau}^{x}: \quad N_{x} S_{\tau}^{+} \longrightarrow N_{x} S_{\tau}^{-},
$$

with the following property: for any normal 1-form field $\boldsymbol{m}$ to $S_{\tau}^{+}$(i.e. a section of $N S_{\tau}^{+}$), we denote the second fundamental form of $S_{\tau}$ with respect to $\boldsymbol{m}$ by $\boldsymbol{K}_{\boldsymbol{S}_{\tau}^{+}}^{+}(\boldsymbol{m}) \equiv \Phi_{\tau,+}^{\star}\left(\nabla^{+} \boldsymbol{m}\right)$. Using $f_{\tau}$ we can map $\boldsymbol{m}$ to a normal 1 -form field $f_{\tau}(\boldsymbol{m})$ to $S_{\tau}^{-}$and calculate the second fundamental form of $S_{\tau}$ with respect to this normal. These two second fundamental forms are required to coincide, i.e.

$$
\boldsymbol{K}_{\boldsymbol{S}_{\tau}}^{+}(\boldsymbol{m})=\boldsymbol{K}_{\boldsymbol{S}_{\tau}}^{-}\left(f_{\tau}(\boldsymbol{m})\right), \quad \forall \text { sections } \boldsymbol{m}: S_{\tau}^{+} \rightarrow N S_{\tau}^{+} .
$$

\section{Consequences of the matching on the FLRW region}

After this reminder on matching conditions, let us now study the matching between a FLRW spacetime and a static spacetime. We start with some definitions which fix our notation and conventions.

Definition 1. Let $\left(\mathcal{M}, g_{\mathcal{M}}\right)$ be a complete, simply connected, three-dimensional Riemannian manifold of constant curvature and $I \subset \mathbb{R}$ be an open interval. A FLRW spacetime $\left(V^{F L}, g^{F L}\right)$ is the manifold $V^{F L}=I \times \mathcal{M}$ endowed with the metric $g^{F L}=-\mathrm{d} t^{2}+a^{2}(t) g_{\mathcal{M}}$, where the so-called scale factor $a(t)$ is a positive $C^{3}$ function on $I$. Moreover, the following conditions are required:

(1) The energy density $\rho$ and pressure $p$ of the cosmological flow satisfy $\rho \geqslant 0, \rho+p \neq 0$.

(2) The expansion $\dot{a} / a$ (where a dot denotes $\mathrm{d} / \mathrm{d} t$ ) vanishes nowhere on $I$.

Remark 1. The energy condition $\rho \geqslant 0$ is used only in proposition 1 in order to exclude some non-compact boundaries for the static region. Thus, this condition could be replaced by spatial compactness of the matching hypersurface. Requiring $\rho \geqslant 0$ is preferable as this holds for most physically reasonable FLRW spacetimes.

Remark 2. The condition $\dot{a} \neq 0$ is made for simplicity and implies no fundamental restriction. Any FLRW spacetime which is not static (an uninteresting case) can be split into open disjoint regions which are either expanding or contracting. The results of this paper apply to each one of those regions independently.

We will denote by $\pi$ the canonical projection from $V^{F L}$ into $\mathcal{M}$ and by $\mathcal{M}_{t}$ the hypersurfaces $t=$ constant.

Definition 2. Let $\left(\Xi, g_{\Xi}\right)$ be a three-dimensional, connected $C^{3}$ Riemannian manifold and $I_{1} \subset \mathbb{R}$ an open interval. The static spacetime $\left(V^{s t}, g^{s t}\right)$ is the manifold $V^{\text {st }}=I_{1} \times \Xi$, with metric $g^{s t}=-D^{2} \mathrm{~d} T^{2}+g_{\Xi}$, where $D$ is a positive $C^{3}$ function on $\Xi$.

We do not assume, a priori, any specific form for $\Xi, g_{\Xi}$ or $D$. The static Killing vector $\partial_{T}$ of this metric will be denoted by $\vec{\xi}$ and the time orientation is chosen so that $\vec{\xi}$ is future directed.

Our aim is to match these two spacetimes across a common boundary. So, we should look for two $C^{3}$ embeddings $\Phi_{+}: \Omega \rightarrow V^{s t}$ and $\Phi_{-}: \Omega \rightarrow V^{F L}$ such that $\Omega^{ \pm} \equiv \Phi_{ \pm}(\Omega)$ 
are boundaries of submanifold-with-boundary $V^{+} \subset V^{s t}$ and $V^{-} \subset V^{F L}$, respectively. Thus, $\Omega^{ \pm}$are embedded $C^{3}$ hypersurfaces without a boundary. The two spacetimes we want to join are $\left(V^{+}, g^{+}\right)$and $\left(V^{-}, g^{-}\right)$, where $g^{+}$is the induced static metric and $g^{-}$is the induced FLRW metric. Being part of a FLRW spacetime, $\left(V^{-}, g^{-}\right)$obviously admits a privileged, future-directed, unit, timelike vector field $\vec{u}$ defined as the fluid velocity of the cosmological flow. Let us define a map $\chi: \Omega^{-} \rightarrow \mathbb{R}$ which assigns to each $x \in \Omega^{-}$the value of the cosmic time $t$ at $x$. Since $\Omega^{-}$is $C^{3}$ it follows that $\chi$ is also $C^{3}$. So, the Morse-Sard theorem (see, e.g., [18]) implies that the set of critical values of $\chi$ has measure zero in $\mathbb{R}$. If $\chi\left(\Omega^{-}\right)$has zero measure itself then it follows, using the fact that $\Omega^{-}$has no boundary, that $\Omega^{-}$is a collection of hypersurfaces of constant time. In that case the resulting matched spacetime does not describe a static region inside a FLRW spacetime. Although this case is not difficult to study, it is notationally cumbersome to incorporate into our results. In order to exclude this, and similar cases with the same problem, we put forward the following definition.

Definition 3. A hypersurface $\Omega^{-}$in a FLRW spacetime will be called generic if and only if the function $\left.\chi \equiv t\right|_{\Omega^{-}}$has no local maximum or minimum on $\Omega^{-}$.

Remark. The geometrical meaning of this definition is that for any point $p \in \Omega^{-}$and for any neighbourhood $U \subset \Omega^{-}$of $p$ there exists a portion of $U$ into the future of $p$ and another portion of $U$ into the past of $p$. In other words, the hypersurface $\Omega^{-}$may not appear or disappear in time, even in a local sense. In particular, hypersurfaces which are tangent to a constant-time hypersurface $\mathcal{M}_{t_{0}}$ on some open neighbourhood are excluded. As mentioned above, nongeneric hypersurfaces can be incorporated into our results but only at a high notational cost. In order to keep the notation reasonably simple we will restrict ourselves to the generic situation. It must be emphasized that causal hypersurfaces are automatically generic according to our definition. Thus, the condition of genericity does not imply any loss of generality from the physical point of view.

For a generic hypersurface, $\chi\left(\Omega^{-}\right)$is necessarily open in $I$. Let us denote by $J$ the set of regular values of $\chi$. The implicit function theorem implies that, for any $\tau \in J$, the pre-image $S_{\tau}^{-} \equiv \chi^{-1}(\tau)=\mathcal{M}_{\tau} \cap \Omega^{-}$is a two-dimensional $C^{3}$ embedded submanifold of $\Omega^{-}$. These surfaces foliate an open subset $\Omega_{0}^{-} \subset \Omega^{-}$. If $\Omega^{-}$is generic then $\Omega_{0}^{-}$is dense.

We shall now impose the constraint matching conditions on the foliation $\left\{S_{\tau}^{-}\right\}$of $\Omega_{0}^{-}$. For all $\tau \in J$, we define $\vec{s}$ as the unit normal vector of $S_{\tau}^{-}$which is tangent to the hypersurface $\mathcal{M}_{\tau}$ and pointing inwards in $V^{-}$. This vector is easily seen to be nowhere tangent to $\Omega_{0}^{-}$.

The following proposition is an important step towards solving our matching problem.

Proposition 1. Let $(V, g)$ be the matching spacetime between a FLRW region $\left(V^{-}, g^{-}\right)$and a static region $\left(V^{+}, g^{+}\right)$across a connected, generic matching hypersurface $\Omega^{-}$. Let $S_{\tau}^{-}$be the foliation of $\Omega_{0}^{-}$defined above. Then, the following geometric properties hold.

(1) $\vec{\xi}$ is orthogonal to each 2-surface $S_{\tau}^{-}$everywhere.

(2) The hyperbolic angle between $\vec{u}$ and $\vec{\xi}$ is constant on each connected component of the 2-surface $S_{\tau}^{-}$.

(3) Each connected component of $S_{\tau}^{-}$is a 2-sphere with the standard metric and it is an umbilical submanifold if $(V, g)$. Furthermore, there exists a spherically symmetric coordinate system $\{t, r, \theta, \phi\}$ in $\left(V^{-}, g^{-}\right)$such that this surface corresponds to $r=$ constant, $t=$ constant.

Remark 1. We assume connectedness of $\Omega^{-}$for convenience. Since the matching conditions are of a local nature, this assumption implies no loss of generality. For an arbitrary matching hypersurface proposition 1 holds for any of its connected components. 
Remark 2. Note that no topological assumptions were made on $\Omega$ except for connectedness. It is remarkable that spatial compactness follows from the matching conditions.

Remark 3. Conclusion (2) of the proposition means, in particular, that the foliation by cosmic time and the foliation by the static time agree on the matching hypersurface. This was the restriction on the times made in [12] and discussed in the introduction. Thus, proposition 1 shows that this condition is not an extra assumption but rather a consequence of the matching.

Remark 4. Conclusion (3) states that each surface $S_{\tau}^{-}$is a coordinate 2-sphere in some spherically symmetric coordinate system. These spheres, however, need not be concentric to each other. In other words, the centre of each $S_{\tau}^{-}$in the FLRW spacetime is still allowed to move arbitrarily with cosmic time.

Proof. Here and in the following (, ) will represent a scalar product with the metric $g$ of the joined spacetime. Let us fix a regular value $\tau_{0}$ of $\chi$ and the corresponding surface $S_{\tau_{0}}^{-}$. For any point $p \in S_{\tau_{0}}^{-}$consider an open neighbourhood $U \subset \Omega_{0}^{-}$of $p$. Denoting by $\boldsymbol{n}$ the normal 1 -form to $\Omega^{-}$, we know that $\boldsymbol{n}(\vec{s}) \neq 0$ everywhere on $U$ (because $\vec{s}$ is transverse to $\Omega_{0}^{-}$). After restricting $U$ if necessary, we can take a pair of vector fields $\vec{e}_{A}(A=1,2)$ on $U$ which are linearly independent at every point and tangent to the foliation $\left\{S_{\tau}^{-}\right\}$. We can complete $\left\{\vec{e}_{A}\right\}$ to a basis $T_{q} V$ by taking the fluid velocity vector $\vec{u}$ and the vector field $\vec{s}$. The Killing vector $\vec{\xi}$ at points in $U$ (remember that we identify $\Omega^{-}$and $\Omega^{+}$after the matching) can be expanded in this basis to give

$$
\left.\vec{\xi}\right|_{U}=D \cosh \beta \vec{u}-D \sinh \beta \cos \alpha \vec{s}+\left.c^{A} \vec{e}_{A}\right|_{U},
$$

where $D$ was introduced before and $\alpha, \beta, c^{A}$ are scalar functions on $U$. Defining $h_{A B}=$ $\left.\left(\vec{e}_{A}, \vec{e}_{B}\right)\right|_{U}$, the functions $c^{A}$ satisfy $c^{A} c^{B} h_{A B}=D^{2} \sinh ^{2} \beta \sin ^{2} \alpha$, so that, in particular, $c^{A}=0$ if either $\alpha$ or $\beta$ vanish. Let us introduce the two vector fields on $U$

$$
\left.\vec{v}_{A}\right|_{U}=[\boldsymbol{n}(\vec{s}-\tanh \beta \cos \alpha \vec{u})] \vec{e}_{A}+\left.\frac{h_{A B} c^{B}}{D \cosh \beta}[\boldsymbol{n}(\vec{s}) \vec{u}-\boldsymbol{n}(\vec{u}) \vec{s}]\right|_{U},
$$

which are tangent to $\Omega$ and orthogonal to $\vec{\xi}$. We now use equation (4), which relates the two Riemann tensors on the matching hypersurface. The Riemann tensor in the FLRW region is

$$
R_{\alpha \beta \lambda \mu}^{-}=\frac{\rho+p}{2}\left[u_{\alpha} u_{\lambda} g_{\beta \mu}^{-}-u_{\alpha} u_{\mu} g_{\beta \lambda}^{-}+u_{\beta} u_{\mu} g_{\alpha \lambda}^{-}-u_{\beta} u_{\lambda} g_{\alpha \mu}^{-}\right]+\frac{\rho}{3}\left(g_{\alpha \lambda}^{-} g_{\beta \mu}^{-}-g_{\alpha \mu}^{-} g_{\beta \lambda}^{-}\right) \text {. }
$$

The geometry of the static region has not yet been fixed, so its Riemann tensor is still arbitrary. However, for any static spacetime and any triad of vectors $\vec{t}_{1}, \vec{t}_{2}, \vec{t}_{3}$ which are orthogonal to $\vec{\xi}$, the following identity holds:

$$
R_{\alpha \beta \lambda \mu}^{+} \xi^{\alpha} t_{1}^{\beta} t_{2}^{\lambda} t_{3}^{\mu}=0
$$

Therefore, contracting (4) with $\xi^{\alpha}, v_{A}^{\beta}, v_{B}^{\lambda}$ and $v_{C}^{\mu}$ we obtain

$$
0=\left.\frac{\rho+p}{2}(\vec{u}, \vec{\xi})\left[\left(\vec{u}, \vec{v}_{B}\right)\left(\vec{v}_{A}, \vec{v}_{C}\right)-\left(\vec{u}, \vec{v}_{C}\right)\left(\vec{v}_{A}, \vec{v}_{B}\right)\right]\right|_{U}
$$

Evaluating the scalar products we obtain

$0=-\left.\frac{\rho+p}{2}(\vec{u}, \vec{\xi})[\boldsymbol{n}(\vec{s}-\tanh \beta \cos \alpha \vec{u})]^{2} \frac{\boldsymbol{n}(\vec{s})}{D \cosh \beta}\left(c_{B} h_{A C}-c_{C} h_{A B}\right)\right|_{U}$,

where $c_{A} \equiv h_{A B} c^{B}$. Using $\rho+p \neq 0$ (and recalling that $\boldsymbol{n}(\vec{s}) \neq 0$ ) we conclude that either $\boldsymbol{n}(\vec{s}-\tanh \beta \cos \alpha \vec{u})=0$ or $c_{B} h_{A C}-c_{C} h_{A B}=0$. The first case is impossible. Indeed, 
suppose that there exists a point $q \in U$ where $\left.\boldsymbol{n}(\vec{s}-\tanh \beta \cos \alpha \vec{u})\right|_{q}=0$. Then, $\vec{v}_{A}$ (9) are not linearly independent at $q$, so that it is not surprising that (12) vanishes. Let us, instead, contract identity (4) with the vectors $\xi^{\alpha}, t_{1}^{\beta}, t_{2}^{\lambda}, t_{1}^{\mu}$, where $\vec{t}_{1}=R^{A} \vec{e}_{A}$ ( $R^{A}$ is any non-zero solution of $\left.R^{A} c_{A}\right|_{q}=0$ ) and $\vec{t}_{2}=\vec{s}-\tanh \beta \cos \alpha \vec{u}$, which is orthogonal to $\vec{\xi}$ at $q$ by construction. The result is

$$
0=\left.\frac{\rho+p}{2}(\vec{u}, \vec{\xi})\left(R^{A} R^{B} h_{A B}\right) \tanh \beta \cos \alpha\right|_{q} .
$$

It follows that $\left.\tanh \beta \cos \alpha\right|_{q}=0$ and therefore $\left.\boldsymbol{n}(\vec{s})\right|_{q}=0$, which is impossible in $U$.

Thus, equation (12) implies $c_{B} h_{A C}-\left.c_{C} h_{A B}\right|_{U}=0$, which is equivalent to $\left.c^{A}\right|_{U}=0$. Hence conclusion (1) of the proposition follows from (8), which becomes

$$
\left.\vec{\xi}\right|_{U}=D \cosh \beta \vec{u}-\left.D \sinh \beta \vec{s}\right|_{U}
$$

To prove the other results, let us denote by $\vec{K}$ the second fundamental form vector (see, e.g., [19]) of the surface $S_{\tau_{0}}^{-}$embedded in $(V, g)$. The constraint matching conditions imply that this vector is well defined on $S_{\tau_{0}}^{-}$(i.e. it is independent of whether it is evaluated from $V^{-}$ or from $\left.V^{+}\right)$. From conclusion (1) we have that $\xi \equiv g(\vec{\xi}, \cdot)$ is a normal 1-form to $S_{\tau_{0}}^{-}$. The second fundamental form $\vec{K}(\xi)$ vanishes in the static region (and hence in the FLRW region) as a consequence of the Killing equations $\nabla_{\alpha}^{+} \xi_{\beta}+\nabla_{\beta}^{+} \xi_{\alpha}=0$. Evaluating this expression from $V^{-}$we obtain, after using (13), $\vec{K}(\boldsymbol{u})-\tanh \beta \vec{K}(s)=0$. The second fundamental form $\vec{K}(\boldsymbol{u})$ of $S_{\tau_{0}}^{-}$in the FLRW region is easy to obtain and reads $\left.\vec{K}(\boldsymbol{u})\right|_{S_{\tau_{0}}^{-}}=\left.(\dot{a} / a) h\right|_{S_{\tau_{0}}^{-}}$, where $\left.h\right|_{S_{\tau_{0}}^{-}}$is the induced metric on $S_{\tau_{0}}^{-}$. Since $\dot{a}$ is nowhere zero by assumption (see definition 1 ), we conclude that $\beta$ is nowhere zero on $S_{\tau_{0}}^{-}$and also that

$$
\left.\vec{K}\right|_{S_{\tau_{0}}^{-}}=\left.\frac{\dot{a}}{a}(\vec{u}+\operatorname{coth} \beta \vec{s}) h\right|_{S_{\tau_{0}}^{-}}
$$

which shows, in particular, that $S_{\tau_{0}}^{-}$is umbilical in the FLRW region (and hence in $(V, g)$ ). Now invoking the Gauss equation (see, e.g., [19]), we obtain

$$
R_{A B C D}^{(2)}\left(\tau_{0}\right)=\left.\left(\frac{\rho}{3}+\frac{\dot{a}^{2}}{a^{2}} \frac{1}{\sinh ^{2} \beta}\right)\left(h_{A C} h_{B D}-h_{A D} h_{B C}\right)\right|_{S_{\tau_{0}^{-}}^{-}},
$$

where $R_{A B C D}^{(2)}\left(\tau_{0}\right)$ is the Riemann tensor of the induced metric of $S_{\tau_{0}}^{-}$. Recalling that $\rho \geqslant 0$, it follows that $S_{\tau_{0}}^{-}$has positive constant curvature. Thus, it is locally isometric to a 2 -sphere with the standard metric. In particular, $\beta$ is constant along each connected component of $S_{\tau_{0}}^{-}$. This proves conclusion (2) of the proposition. In order to show (3) we use the following well known result (see, e.g., [20]).

A connected, complete and orientable surface of positive constant curvature is isometric to $S^{2}$ (the 2-sphere with standard metric).

We only need to check that $S_{\tau_{0}}^{-}$is orientable and complete. Completeness follows from the fact that $S_{\tau_{0}}^{-}$is a closed subset of $\mathcal{M}_{\tau_{0}}$, which is a complete space. Orientability can be proven as follows. $V^{F L}$ is an oriented space and therefore $\Omega^{-}$(which is the boundary of the submanifold-with-boundary $V^{-}$) is a three-dimensional submanifold of $V^{F L}$ with a canonical orientation (see, e.g., theorem 5.3.36 of [21]). Consider the subset $\left\{p \in \Omega^{-} ; \chi(p) \geqslant \tau_{0}\right\}$. This is a submanifold-with-boundary of $\Omega$ with boundary $S_{\tau_{0}}^{-}$. Applying theorem 5.3.36 of [21] once again, we conclude that $S_{\tau_{0}}^{-}$is orientable, as claimed. So, each connected component of $S_{\tau_{0}}^{-}$is isometric to a 2 -sphere. 
It only remains to obtain the coordinate form of $S_{\tau_{0}}^{-} . \mathcal{M}_{\tau}$ is an umbilical hypersurface in $\left(V^{F L}, g^{F L}\right)$. Consequently, $S_{\tau_{0}}^{-}$is also umbilical in $\mathcal{M}_{\tau_{0}}$. Using the fact that $\mathcal{M}_{\tau_{0}}$ is isometric to $\mathbb{R}^{3}, S^{3}$ or $\mathbb{H}^{3}$ (with the standard metric), a well known result (see, e.g., theorem 20 , chapter 7 in [22]) states that each connected component of $S_{\tau_{0}}^{-}$must be an $r=$ constant surface in some spherical coordinate system for $\mathcal{M}_{\tau_{0}}$. This proves conclusion (3) of the proposition.

Proposition 1 shows that the matching hypersurface $\Omega^{-}$corresponds to a collection of 2 -spheres moving on spacetime and with arbitrarily changing radius. Moreover, these 2spheres cannot merge. Indeed, if two 2-spheres merge touching each other at one point, then the hypersurface $\Omega^{-}$would cease to be $C^{3}$. Alternatively, two 2-spheres might merge at all points simultaneously. This would imply that $\chi$ has either a maximum or a minimum. This is excluded by our condition that $\Omega^{-}$should be generic. Note that this argument shows, in particular, that no saddle points of $\chi$ may exist. Thus the following corollary holds:

Corollary 1. Let $\Omega^{-}$be the hypersurface in proposition 1. Then $\chi$ has no critical points, i.e. $\left\{S_{\tau}^{-}\right\}$defines a foliation on the whole of $\Omega^{-}$.

Note, in particular, that when $\Omega^{-}$is connected then each element of the foliation $\left\{S_{\tau}^{-}\right\}$is connected as well. Another obvious consequence of corollary 1 is that $\Omega_{0}^{-}=\Omega^{-}$.

In proposition 1 only the constraint matching conditions were used. In order to analyse the remaining conditions, let us define $\vec{m}$ as a vector field tangent to $\Omega^{-}$and orthogonal to the foliation $\left\{S_{\tau}^{-}\right\}$of $\Omega^{-}$. Since all points in $\Omega^{-}$are regular, we have $(\vec{u}, \vec{m}) \neq 0$ everywhere. This allows us to fix $\vec{m}$ uniquely by requiring $\left.(\vec{m}, \vec{u})\right|_{\Omega^{-}}=-1$. By definition, $\vec{m}$ is a $C^{2}$ vector field which can be decomposed as

$$
\left.\vec{m}\right|_{\Omega^{-}}=\vec{u}+\left.\mu \vec{s}\right|_{\Omega^{-}},
$$

where $\mu$ is a $C^{2}$ scalar function on $\Omega^{-}$. The normal 1 -form $\boldsymbol{n}$ can be decomposed as $\left.\boldsymbol{n}\right|_{\Omega^{-}}=\mu \boldsymbol{u}+\left.\boldsymbol{s}\right|_{\Omega^{-}}$, where $\boldsymbol{u}=g^{F L}(\vec{u}, \cdot)$ and $s=g^{F L}(\vec{s}, \cdot)$. We also define $\vec{w}$ as

$$
\left.\vec{w}\right|_{\Omega^{-}}=-D \sinh \beta \vec{u}+\left.D \cosh \beta \vec{s}\right|_{\Omega^{-}} .
$$

The following simple lemma has interesting geometrical consequences.

Lemma 1. The static Killing vector $\vec{\xi}$ is nowhere tangent to $\Omega$.

Proof. Fix a point $p \in \Omega^{-}$and consider the open neighbourhood $U$ of $p$ used in proposition 1 . Contracting identity (4) with $\xi^{\alpha}, e_{A}^{\beta}, w^{\lambda}, e_{B}^{\mu}$ and using $R_{\alpha \beta \lambda \mu}^{+} \xi^{\alpha} e_{A}^{\beta} w^{\lambda} e_{C}^{\mu}=0$ (which holds because $\vec{w}$ is orthogonal to $\vec{\xi}$ ), we obtain

$$
\left.\boldsymbol{n}(\vec{\xi}) \boldsymbol{n}(\vec{w}) B_{\beta \mu} \mathrm{e}_{A}^{\beta} \mathrm{e}_{B}^{\mu}\right|_{U}=\left.\frac{\rho+p}{2} D^{2} \sinh \beta \cosh \beta h_{A B}\right|_{U} .
$$

The tensor $B_{\alpha \beta}$ is at least $C^{0}$ on $U$ and $\tanh \beta$ is nowhere zero on $U$ (from proposition 1). Therefore, we must have $\left.n(\vec{\xi})\right|_{U} \neq 0$ and the lemma follows.

This result tells us that the matching hypersurface cannot be locally static anywhere. This will be used later in order to determine the geometry of the static region. A more technical, but useful, consequence of lemma 1 is that $\vec{\xi}$ defines a rigging vector on $\Omega$. This will simplify our calculations considerably. Indeed, although the matching conditions are independent of the choice of rigging, the complexity of the calculations may depend on which rigging is used.

Recalling that only the symmetric part of (3) needs to be considered, the Killing equations for $\vec{\xi}$ and expression (13) imply

$$
\Phi_{-}^{\star}\left[\nabla_{(\alpha}^{-}\left(D \cosh \beta u_{\gamma)}\right)-\nabla_{(\alpha}^{-}\left(D \sinh \beta s_{\gamma)}\right)\right]=0 .
$$


This expression is remarkable because, except for the scalar function $D$, only quantities from the FLRW region are involved. To analyse it further we need the covariant derivatives of $\boldsymbol{u}$ and of $s$. The first one is trivial, $\nabla_{\alpha}^{-} u_{\beta}=(\dot{a} / a)\left(g_{\alpha \beta}^{-}+u_{\alpha} u_{\beta}\right)$, and the second one is given in the following lemma.

Lemma 2. The covariant derivative of s reads

$$
\left.\nabla_{\alpha} s_{\beta}\right|_{\Omega^{-}}=\frac{\dot{a}}{a} u_{\beta} s_{\alpha}+\frac{\dot{a}}{a} \operatorname{coth} \beta h^{A B} e_{A \alpha} e_{B \beta}+\left.h^{A B} \vec{e}_{A}(\mu) e_{B \beta} u_{\alpha}\right|_{\Omega^{-}} .
$$

Proof. Take $p \in \Omega^{-}$and choose the neighbourhood $U$ of $p$ we have been using throughout. Since $\vec{s}$ is unit, orthogonal to $\vec{u}$ and expression (14) holds, we easily find

$$
\left.\nabla_{\alpha} s_{\beta}\right|_{U}=\frac{\dot{a}}{a}\left(u_{\beta} s_{\alpha}+\operatorname{coth} \beta h^{A B} e_{A \alpha} e_{B \beta}\right)+v_{\beta} u_{\alpha}+\left.b_{\beta} s_{\alpha}\right|_{U},
$$

where $\vec{v}$ and $\vec{b}$ are vectors tangent to $\left\{S_{\tau}^{-}\right\}$. Taking into account that $\left.\vec{s}\right|_{S_{\tau}^{-}}$is invariant under the isometry group $S O(3)$ acting on $S_{\tau}^{-}$, we conclude that $\vec{b}=0$. So, we only need to determine $\vec{v}$. To do that, recall that the normal 1-form $\boldsymbol{n} \operatorname{reads} \boldsymbol{n}=\mu \boldsymbol{u}+\boldsymbol{s}$. Since $\boldsymbol{n}$ is integrable, it must satisfy $\boldsymbol{n} \wedge \mathrm{d} \boldsymbol{n}=0$. Using $\mathrm{d} \boldsymbol{u}=0$ and expression (20) this equation becomes $\left.s \wedge \boldsymbol{u} \wedge(\mathrm{d} \mu-\boldsymbol{v})\right|_{U}=0$. This implies $\mathrm{d} \mu=\boldsymbol{v}+r_{1} \boldsymbol{u}+r_{2} s$, and hence $\left.\left(\vec{v}, \vec{e}_{A}\right)\right|_{U}=\vec{e}_{A}(\mu)$, which proves the lemma.

We can now impose the matching condition (19).

Lemma 3. The matching condition (19) is satisfied on $\Omega^{-}$if and only if the following two conditions hold:

(1) there exists a nowhere-vanishing function $\Delta$ on $\Omega^{-}$which is constant on each 2-sphere $S_{\tau}^{-}$such that $\left.D\right|_{\Omega^{-}}=\left.\Delta^{-1}(\cosh \beta+\mu \sinh \beta)\right|_{\Omega^{-}}$;

(2) the quantity $Z \equiv \mu /$ a satisfies the following partial differential equation on $\Omega^{-}$:

$$
\vec{m}(Z)=\frac{\dot{a} Z^{2}}{\tanh \beta}+Z\left[\frac{\dot{\Delta}}{\Delta}-\frac{2 \cosh ^{3} \beta}{\sinh \beta} \vec{m}(\tanh \beta)\right]+\frac{\dot{\Delta}}{\Delta a \tanh \beta}-\frac{2}{a} \vec{m}(\beta) .
$$

Proof. Let us contract (19) with $e_{A}^{\alpha} m^{\beta}$ and $m^{\alpha} m^{\beta}$, and use lemma 2 (the contraction with $e_{A}^{\alpha} e_{B}^{\beta}$ is identically satisfied because the constraint matching conditions have already been imposed). The first of these contractions gives

$$
(1+\mu \tanh \beta) \vec{e}_{A}(D)=D \tanh \beta \vec{e}_{A}(\mu) .
$$

Let us define $\Delta \equiv(\cosh \beta+\mu \sinh \beta) / D$ on $\Omega^{-}$. From the fact that $\boldsymbol{n}(\vec{w}) \neq 0$ everywhere (see the proof of lemma 1 ), we find $(1+\mu \tanh \beta) \neq 0$ on $\Omega^{-}$. Hence $\Delta$ cannot vanish. Substituting $D$ in terms of $\Delta$ in (22) we obtain $\left.\vec{e}_{A}(\Delta)\right|_{\Omega^{-}}=0$, which proves the first part of the lemma. To arrive at (21) we contract (19) with $m^{\alpha} m^{\beta}$. After a short calculation we obtain

$$
\vec{m}(D)=\frac{1}{\Delta}(\sinh \beta+\mu \cosh \beta)\left[\frac{\dot{a}}{a} \mu-\vec{m}(\beta)\right] .
$$

Inserting the expression for $D$ in terms of $\Delta$ and the redefinition $\mu=Z a$ we readily obtain (21).

The next task is trying to solve the PDE (21). To that end a suitable coordinate system in the FLRW region has to be introduced. We know that $\Omega^{-}$is foliated by a collection of 
2-surfaces. Since $\Omega^{-}$is $C^{3}$, the centre of each one of these surfaces moves along some $C^{3}$ path $\sigma_{1}: I_{2} \subset \mathbb{R} \rightarrow V^{F L}$, where $I_{2}$ is an open subinterval of $I$. Using the fact that $\vec{m}$ is future directed, it follows that $g^{F L}\left(\dot{\sigma}_{1}, \vec{u}\right)<0$ and hence we can project $\sigma_{1}$ onto the $\mathcal{M}$ factor of $V^{F L}=I \times \mathcal{M}$ to define a $C^{3}$ map $\sigma: I_{2} \rightarrow \mathcal{M}$. We now use the fact that $\mathcal{M}$ is a maximally symmetric space and hence homogeneous. Let $G$ be the isometry group of $\mathcal{M}$, and fix a point $p=\sigma\left(t_{0}\right)$ for some $t_{0} \in I_{2}$. From the homogeneity of $\mathcal{M}$, there exists, for each $t \in I_{2}$, an element $\tilde{\gamma}(t) \in G$ which maps $p$ into $\sigma(t)$. Let us define $\gamma(t) \equiv \tilde{\gamma}\left(t+t_{0}\right)$ and choose $\gamma(0)=e$, where $e$ is the identity of $G$. Given that the isotropy group of $G$ is non-trivial, there are still many possible choices for $\gamma(t)$. We can fix it uniquely as follows. Since $\mathcal{M}$ is a symmetric space, the Lie algebra of $G$ admits a canonical decomposition (see, e.g., [23]) $\mathfrak{g}=\mathfrak{h} \oplus \mathfrak{l}$, where $\mathfrak{h}$ is the isotropy algebra at $p$ and $\mathfrak{l}$ is the subspace of the so-called transvections at $p$. For each $t$ satisfying $t+t_{0} \in I_{2}$, consider the map $U_{t}(\lambda) \equiv \gamma(t+\lambda) \gamma^{-1}(t)$ which is a $C^{3}$ curve in $G$ passing through $e$. Its tangent vector at the identity defines an element of $\mathfrak{g}$. Only the component in $\mathfrak{l}$ moves the point $\sigma(t)$ (the component in $\mathfrak{h}$ corresponds to the isotropy group at $\sigma(t))$. Using this fact, it is not difficult to prove that $\gamma(t)$ can be uniquely fixed by demanding that the tangent vector of $U_{t}(\lambda)$ at $\lambda=0$ has vanishing component in $\mathfrak{h}$. We assume this choice from now on. Then, a coordinate system in the FRLW region adapted to the matching hypersurface can be constructed as follows. Take a spherically symmetric coordinate system $\{r, \theta, \phi\}$ of $\mathcal{M}$ centred at $p$. The line-element of $\mathcal{M}$ takes the standard form $\mathrm{d} s^{2}=\mathrm{d} r^{2}+\Sigma^{2}(r, \epsilon)\left(\mathrm{d} \theta^{2}+\sin ^{2} \theta \mathrm{d} \phi^{2}\right)$, where

$$
\Sigma(r, \epsilon)= \begin{cases}\sin r & \text { if } \epsilon=1, \\ r & \text { if } \epsilon=0, \\ \sinh r & \text { if } \epsilon=-1 .\end{cases}
$$

Given any $t$ satisfying $t+t_{0} \in I_{2}$ we define a unique spherically symmetric coordinate system of $\mathcal{M}_{t}$ centred at $\sigma(t)$ as follows. The coordinate values of a point $q \in \mathcal{M}_{t}$ are taken to be the same as the spherically symmetric coordinates $\{r, \theta, \phi\}$ of the point $\gamma^{-1}(t)(\pi(q)$ ) (recall that $\pi$ is the canonical projection of $V^{F L}$ into $\left.\mathcal{M}\right)$. The fourth coordinate of $q$ is just the value of the cosmic time at $q$. In the appendix we describe how to obtain the FLRW metric in this coordinate system. The result is

$\mathrm{d} s^{2}=-\mathrm{d} t^{2}+a^{2}(t)\left[\left(\mathrm{d} r+\eta_{t}^{r} \mathrm{~d} t\right)^{2}+\Sigma^{2}(r, \epsilon)\left(\left(\mathrm{d} \theta+\eta_{t}^{\theta} \mathrm{d} t\right)^{2}+\sin ^{2} \theta\left(\mathrm{d} \phi+\eta_{t}^{\phi} \mathrm{d} t\right)^{2}\right)\right]$,

where $\vec{\eta}_{t}=h^{i}(t) \vec{\eta}_{i}(i=1,2,3), h^{i}(t)$ are $C^{3}$ real functions on $I_{2}$, satisfying $h^{2}\left(t_{0}\right)=h^{3}\left(t_{0}\right)=$ 0 and $\vec{\eta}_{i}$ are the transvection Killing vector fields

$$
\begin{aligned}
& \vec{\eta}_{1}=\cos \theta \frac{\partial}{\partial r}-\frac{\Sigma_{, r}}{\Sigma} \sin \theta \frac{\partial}{\partial \theta}, \\
& \vec{\eta}_{2}=\sin \theta \cos \phi \frac{\partial}{\partial r}+\frac{\Sigma_{, r}}{\Sigma}\left(\cos \theta \cos \phi \frac{\partial}{\partial \theta}-\frac{\sin \phi}{\sin \theta} \frac{\partial}{\partial \phi}\right), \\
& \vec{\eta}_{3}=\sin \theta \sin \phi \frac{\partial}{\partial r}+\frac{\Sigma_{, r}}{\Sigma}\left(\cos \theta \sin \phi \frac{\partial}{\partial \theta}+\frac{\cos \phi}{\sin \theta} \frac{\partial}{\partial \phi}\right) .
\end{aligned}
$$

In this coordinate system, each connected component of $\Omega^{-}$takes the simple form $r-r(t)=0$ where $r(t)$ is a $C^{3}$ non-negative real function. The 2-sphere $S_{\tau}^{-}$is defined just by $t=\tau$ and $r=r(\tau)$. Using (15) and the fact that $\rho=\left(\dot{a}^{2}+\epsilon\right) / a^{2}$ we can easily obtain an explicit formula for $\beta(t)$, namely,

$$
\left.\tanh \beta\right|_{S_{t}^{-}}=\left.\epsilon_{1} \frac{\Sigma \dot{a}}{\Sigma_{, r}}\right|_{r=r(t)},
$$


where $\epsilon_{1}= \pm 1$. This sign depends on whether $\vec{s}$ points radially outwards or inwards in the sphere $S_{t}^{-}$. Recall that $\vec{s}$ was defined to point inwards in $V^{-}$. So, $\epsilon_{1}$ determines which one of the two open sets in $V^{F L}$ separated by $\Omega^{-}$is chosen to perform the matching with the static region. Specifically, $\epsilon_{1}=+1$ corresponds to $V^{-}=\{r \geqslant r(t)\}$ and $\epsilon_{1}=-1$ to $V^{-}=\{r \leqslant r(t)\}$. The explicit expressions for $\vec{u}, \vec{s}$ and $\vec{m}$ in the coordinate system (25) are easily obtained to be

$\vec{s}=\epsilon_{1} \frac{1}{a} \frac{\partial}{\partial r}, \quad \vec{u}=\frac{\partial}{\partial t}-\left(\eta_{t}^{r} \frac{\partial}{\partial r}+\hat{\eta}_{t}^{\theta} \frac{\partial}{\partial \theta}+\hat{\eta}_{t}^{\phi} \frac{\partial}{\partial \phi}\right), \quad \vec{m}=\vec{u}+\epsilon_{1} a\left(\dot{r}+\eta_{t}^{r}\right) \vec{s}$,

where $\left.\hat{\eta}_{t}^{i} \equiv \eta_{t}^{i}\right|_{r=r(t)}$. Thus, we have $Z=\mu / a=\epsilon_{1}\left(\dot{r}+\eta_{t}^{r}\right)$ (no hat is needed on $\eta_{t}^{r}$ because this object does not depend on $r$ ). We can now impose the partial differential equation (21). Inserting the expression for $Z$ above, this equation becomes

$\dot{h}^{i} \eta_{i}^{r}=h^{i} h^{j}\left(\hat{\eta}_{i}^{\theta} \frac{\partial}{\partial \theta} \eta_{j}^{r}+\hat{\eta}_{i}^{\phi} \frac{\partial}{\partial \phi} \eta_{j}^{r}+\left(\left.\frac{\Sigma_{, r}}{\Sigma}\right|_{r=r(t)}\right) \eta_{i}^{r} \eta_{j}^{r}\right)+X_{1}(t) h^{i} \eta_{i}^{r}+X_{2}(t)$,

where $X_{1}$ and $X_{2}$ are

$$
\begin{aligned}
& X_{1}=\frac{2 \epsilon_{1} \dot{a} \dot{r}}{\tanh \beta}+\frac{\dot{\Delta}}{\Delta}-\frac{2 \cosh \beta}{\sinh \beta} \dot{\beta}, \\
& X_{2}=-\ddot{r}+\frac{\epsilon_{1} \dot{a} \dot{r}^{2}}{\tanh \beta}+\frac{\dot{\Delta}}{\Delta}\left(\dot{r}+\frac{\epsilon_{1}}{a \tanh \beta}\right)-2\left(\frac{\epsilon_{1}}{a}+\frac{\dot{r}}{\tanh \beta}\right) \dot{\beta} .
\end{aligned}
$$

Using the Killing equations for $\vec{\eta}_{i}$ it is not difficult to rewrite equation (29) in the following, more elegant form:

$$
2 \dot{h}^{i} \eta_{i}^{r}+\left.h^{i} h^{j} \Sigma^{2} \partial_{r}\left(\Sigma^{-2}\left(\vec{\eta}_{i}, \vec{\eta}_{j}\right)\right)\right|_{r=r(t)}=2 X_{1} h^{i} \eta_{i}^{r}+2 X_{2} .
$$

Furthermore, using the explicit expressions (26), the following relation holds $\partial_{r}\left[\Sigma^{-2}\left(\vec{\eta}_{i}, \vec{\eta}_{j}\right)\right]=-2 \Sigma_{, r} \Sigma^{-3} \delta_{i j}$, so that the equation takes the form

$$
\dot{h}^{i} \eta_{i}^{r}=h^{i} h^{j} \delta_{i j}\left(\left.\frac{\Sigma_{, r}}{\Sigma}\right|_{r=r(t)}\right)+X_{1} h^{i} \eta_{i}^{r}+X_{2} .
$$

Since $\left\{\eta_{i}^{r}, 1\right\}$ are functionally independent of $\Omega^{-}$we obtain $\dot{h}^{i}=X_{1} h^{i}, i=1,2,3$. From the initial conditions $h^{2}\left(t_{0}\right)=0$ and $h^{3}\left(t_{0}\right)=0$ and the uniqueness of the solution we conclude that $h^{2}(t)=h^{3}(t)=0$, so that only the function $f(t) \equiv h^{1}(t)$ remains. Thus $\vec{\eta}_{t}=f(t) \vec{\eta}_{1}$ and the line-element becomes

$$
\begin{aligned}
\mathrm{d} s^{2}=-\mathrm{d} t^{2}+ & a^{2}(t)\left[(\mathrm{d} r+f(t) \cos \theta \mathrm{d} t)^{2}+\left(\Sigma(r, \epsilon) \mathrm{d} \theta-f(t) \Sigma_{, r}(r, \epsilon) \sin \theta \mathrm{d} t\right)^{2}\right. \\
& \left.+\Sigma^{2}(r, \epsilon) \sin ^{2} \theta \mathrm{d} \phi^{2}\right] .
\end{aligned}
$$

The functions $f(t)$ and $\Delta(t)$ solve the ordinary differential equations

$$
\dot{f}=X_{1} f,\left.\quad f^{2} \frac{\Sigma_{, r}}{\Sigma}\right|_{r=r(t)}+X_{2}=0,
$$

with $X_{1}$ and $X_{2}$ given in (30). Thus (see the appendix), the motion of the centre of the 2-spheres is parallel to the axis of symmetry defined by $\cos \theta=0$. Consequently, the curve $\sigma(t)$ is a geodesic of $\mathcal{M}$ (not necessarily affinely parametrized) and its speed is $\dot{\sigma}(t)=f(t)$. Note that this is not the velocity of the worldline of the centre of the sphere in $V^{F L}$. The tangent vector $\vec{v}$ of this curve satisfies $g^{F L}(\vec{v}, \vec{v})=a^{2}(t) f^{2}(t)-1$, which may be timelike (if $|f(t)|<1 / a(t)$ ), null (if $|f(t)|=1 / a(t)$ ) or spacelike (if $|f(t)|>1 / a(t)$ ). Thus the motion of the centre of the static region can perfectly well be superluminal. This might have interesting applications in relation with the warp drive travel put forward by Alcubierre in [24], but this will not be analysed here any further. 


\section{Geometry of the static region}

Up to now, the static geometry has not been specified at all. In all our results above, we assumed that the joined spacetime $(V, g)$ exists and we found consequences on the matching hypersurface in the FLRW side. In this section, we concentrate on the static region. Specifically, we determine completely the full Riemann tensor of the static region in some neighbourhood of the matching hypersurface. This will be done without introducing any coordinate system. Instead, we use a geometrically preferred tetrad. This gives geometrically more transparent expressions. In the next section where we state and prove theorem 1 we shall also obtain the explicit form of the static metric in suitably defined coordinates.

Recall that $V^{+}$is a submanifold-with-boundary of $V^{s t}=I_{1} \times \Xi$ and that the Killing vector $\vec{\xi}$ is tangent to the $I_{1} \subset \mathbb{R}$ factor. Define $\Pi: V^{s t} \rightarrow \Xi$ as the canonical projection along the orbits of the Killing vector $\vec{\xi}$. In lemma 1 we proved that $\vec{\xi}$ is nowhere tangent to $\Omega^{+}$. This means that the restriction of $\Pi$ on $\Omega^{+}$is a diffeomorphism between $\Omega^{+}$and $\Pi\left(\Omega^{+}\right) \subset \Xi$. In particular, any orthonormal basis at a point $p \in \Omega^{+}$, can be uniquely extended to an orthonormal tetrad along the orbit of the static Killing passing through $p$. Hence, an orthonormal tetrad at $\Omega^{+}$induces an orthonormal tetrad on $\mathcal{U} \equiv I_{1} \times \Pi\left(\Omega^{+}\right)$. We choose the tetrad on $\Omega^{+}$as follows. Since each connected component of $\Omega^{+}$is foliated by 2 -spheres, we can take $\left\{\vec{e}_{A}\right\}$ to be a pair of orthonormal vector fields tangent to each $S_{\tau}^{-}$. They are defined everywhere except for a pair of antipodal points (a north and a south pole) where one of them diverges and the other one vanishes. As usual, we exclude the north and south poles from our discussion (to cover them we would need to use two patches on each 2-sphere, but since this is standard it will not be discussed further). On $\Omega^{-}$we also introduced the vector field $\vec{w}$ (17), which is orthogonal to $\left\{S_{\tau}\right\}$, orthogonal to $\vec{\xi}$ and with norm $(\vec{w}, \vec{w})=-(\vec{\xi}, \vec{\xi})=D^{2}$. Thus, an orthonormal tetrad for every point in $\Omega^{+}$is constructed by taking $\vec{e}_{0}=D^{-1} \vec{\xi}, \vec{e}_{1}=D^{-1} \vec{w}$ and $\vec{e}_{A}$. Its dual frame is denoted by $\left\{\theta^{\alpha}\right\}$. This tetrad can be extended to $\mathcal{U} \subset V^{s t}$ by Lie transport along $\vec{\xi}$. The following proposition gives the Riemann tensor of the static region in terms of this tetrad.

Proposition 2. Assume that the static spacetime $\left(V^{s t}, g^{s t}\right)$ can be matched to a FLRW spacetime through a generic boundary. Then, the metric $g^{\text {st }}$ restricted to the neighbourhood $\mathcal{U}$ defined above has the following properties.

(1) The energy-momentum tensor $T^{\text {st }}$ of $g^{\text {st }}$ reads

$$
T^{s t}=\rho^{s t} \theta^{0} \otimes \theta^{0}+p_{r}^{s t} \theta^{1} \otimes \theta^{1}+p_{t}^{s t}\left(\theta^{2} \otimes \theta^{2}+\theta^{3} \otimes \theta^{3}\right),
$$

where the scalars $\rho^{s t}$, $p_{r}^{s t}$ and $p_{t}^{s t}$ are

$$
\begin{aligned}
\rho^{s t}=\frac{\rho \mu-p \tanh \beta}{\mu+\tanh \beta}, \quad p_{r}^{s t}=\frac{p-\rho \mu \tanh \beta}{1+\mu \tanh \beta}, & \\
p_{t}^{s t}=\frac{3 p-\rho}{6}+ & \frac{\tanh \beta\left(2 h^{A B} \vec{e}_{A}(\mu) \vec{e}_{B}(\mu)-(\rho+p)\left(\mu^{2}-1\right)\right)}{2(\mu+\tanh \beta)(1+\mu \tanh \beta)} \\
+ & {\left[-\ddot{\beta}+\frac{\dot{\Delta}}{\Delta}\left(\frac{\dot{a}}{a} \frac{1}{\tanh \beta}+\dot{\beta}\right)-2 \dot{\beta} \frac{\dot{a}}{a}\left(1+\frac{\mu}{\tanh \beta}\right)\right.} \\
+ & \left.\left(\frac{\ddot{a}}{a}+\mu \frac{\dot{a}^{2}}{a^{2} \tanh \beta}\right)\right]\left[\cosh ^{2} \beta(\mu+\tanh \beta)(1+\mu \tanh \beta)\right]^{-1},
\end{aligned}
$$

and $\rho \equiv 3\left(\dot{a}^{2}+\epsilon\right) / a^{2}$ and $p \equiv\left(-2 a \ddot{a}-\dot{a}^{2}-\epsilon\right) / a^{2}$ are the density and pressure of the FLRW spacetime. 
(2) The Petrov type of $g^{s t}$ is $D$, the double principal null directions are $\vec{e}_{0} \pm \vec{e}_{1}$ and the only non-vanishing Weyl spin coefficient in the null basis canonically associated with $\left\{\vec{e}_{\alpha}\right\}$ is

$$
\Psi_{2}=\frac{1}{6}\left[p-p_{t}^{s t}-\frac{\left(\mu^{2}-1\right)(\rho+p) \tanh \beta}{(1+\mu \tanh \beta)(\mu+\tanh \beta)}\right] .
$$

Remark. The Riemann tensor of the static region has many of the properties of a Riemann tensor in a spherically symmetric spacetime. First, $\Omega^{+}$is foliated by 2 -spheres and the tetrad $\left\{\theta^{\alpha}\right\}$ is adapted to this foliation. Furthermore, the energy-momentum tensor is diagonal in this tetrad and the two eigenvalues corresponding to the directions tangent to the spheres coincide. Moreover, the Petrov type is D and the principal null-directions are orthogonal to the 2-spheres. Thus, the static geometry is very close to spherically symmetric (although not exactly spherically symmetric in general, as we shall see).

Proof. The basic idea is to use equation (4). However, this gives not enough information and it must be complemented with some other expression. We use the following identity (which follows easily from $\nabla_{\alpha}^{+} \nabla_{\beta}^{+} \xi_{\delta}=\xi^{\gamma} R_{\gamma \alpha \beta \delta}^{+}$) valid in any spacetime with a static Killing vector with norm $\xi^{\alpha} \xi_{\alpha}=-D^{2}$ :

$$
\nabla_{\alpha}^{+} \nabla_{\beta}^{+} D^{2}-2 \nabla_{\alpha}^{+} \xi^{\mu} \nabla_{\mu}^{+} \xi_{\beta}=2 \xi^{\mu} \xi^{\nu} R_{\mu \alpha \nu \beta}^{+} .
$$

Let us start with expression (4). The only component of this identity which we did not analyse yet is the contraction with $\xi^{\alpha}, w^{\beta}, e_{A}^{\lambda}$ and $w^{\mu}$. After a calculation we obtain

$$
\left.B_{\alpha \lambda}\left[\boldsymbol{n}(\vec{w}) \xi^{\alpha}-\boldsymbol{n}(\vec{\xi}) w^{\alpha}\right] \mathrm{e}_{A}^{\lambda}\right|_{\Omega^{+}}=0 .
$$

The vector field in brackets is nowhere zero on $\Omega^{+}$, orthogonal to the 2 -spheres $S_{\tau}^{-}$and tangent to the hypersurface $\Omega^{+}$. So, $B_{\alpha \lambda} m^{\alpha} e_{A}^{\lambda}=0$ follows. Combining this result with (18) and recalling that $\boldsymbol{n}(\vec{\xi})$ and $\boldsymbol{n}(\vec{w})$ are nowhere zero, we easily obtain

$$
\left.B_{\alpha \beta}\right|_{\Omega^{+}}=\frac{\rho+p}{2} \frac{D^{2} \sinh \beta \cosh \beta}{\boldsymbol{n}(\vec{\xi}) \boldsymbol{n}(\vec{w})} e_{A \alpha} e_{B} h^{A B}+Q m_{\alpha} m_{\beta}+K_{\alpha} n_{\beta}+K_{\beta} n_{\alpha},\left.\right|_{\Omega^{+}}
$$

where $Q$ and $K_{\alpha}$ are unknown functions on $\Omega^{+}$. Since $B_{\alpha \beta}$ is defined up to the transformation (5), we can put $K_{\alpha}=0$ without loss of generality. So, only $Q$ remains to be evaluated. We now invoke identity (33). Contracting it with $m^{\alpha}, m^{\beta}$ we obtain

$$
2 \xi^{\mu} m^{\alpha} \xi^{\nu} m^{\beta} R_{\mu \alpha \nu \beta}^{+}=2 D \vec{m}(\vec{m}(D))+2 D^{2}\left(m^{\alpha} \nabla_{\alpha}^{+} e_{0}^{\mu}\right)\left(m^{\beta} \nabla_{\beta}^{+} e_{0 \mu}\right)-\left.2 D\left(\nabla_{\vec{m}}^{+} \vec{m}\right)^{\beta} \nabla_{\beta}^{+} D\right|_{\Omega^{+}} .
$$

The first two terms on the right-hand side involve only tangent derivatives to $\Omega^{+}$and can, therefore, be evaluated. Only the third term requires further analysis. We can evaluate it from the FLRW side of $V$. Using (16) and lemma 2 we obtain

$$
\left.\nabla_{\vec{m}}^{-} \vec{m}\right|_{\Omega^{-}}=\left(\mu \frac{\dot{a}}{a}+\vec{m}(\mu)\right) \vec{s}+\mu^{2} \vec{u}-\left.\mu \vec{e}_{A}(\mu) h^{A B} \vec{e}_{B}\right|_{\Omega^{-}} .
$$

This vector is, in general, transverse to $\Omega$. Nevertheless, we only need to know its action on $D$, which is constant along $\vec{\xi}$. So, we decompose $\vec{s}$ and $\vec{u}$ on $\Omega$ in terms of $\vec{m}$ and $\vec{\xi}$ to obtain

$$
\vec{s}=\frac{\cosh \beta \vec{m}-D^{-1} \vec{\xi}}{\mu \cosh \beta+\sinh \beta}, \quad \vec{u}=\frac{\sinh \beta \vec{m}+\mu D^{-1} \vec{\xi}}{\mu \cosh \beta+\sinh \beta} .
$$


Inserting this expressions into (36) and using $\vec{\xi}(D)=0$ we can then evaluate (35). After a somewhat long calculation we find

$$
\begin{aligned}
& R_{\mu \alpha \nu \beta}^{+} \xi^{\mu} m^{\alpha} \xi^{v} m^{\beta}=\frac{D(\mu \cosh \beta+\sinh \beta)}{\Delta}\left[\sinh \beta \cosh \beta \vec{e}_{A}(\mu) \vec{e}_{B}(\mu) h^{A B}+\frac{\dot{a}}{a} \vec{m}(\mu)\right. \\
&\left.+\mu\left(\frac{\ddot{a}}{a}-\frac{\dot{a}^{2}}{a^{2}}\right)-\ddot{\beta}-\frac{\dot{\Delta}}{\Delta}\left(\mu \frac{\dot{a}}{a}-\dot{\beta}\right)\right]\left.\right|_{\Omega^{+}},
\end{aligned}
$$

which allows us to determine $Q$ and therefore the full static Riemann tensor on $\Omega^{+}$. Being the tetrad $\left\{\theta^{\alpha}\right\}$ Lie-constant along $\vec{\xi}$, the resulting expression for $R_{\mu \alpha \nu \beta}^{+}$is valid in the whole of $\mathcal{U}$. Splitting the Riemann tensor into the Weyl tensor and the Einstein tensor $G_{\alpha \beta}$ and using Einstein's equations $G_{\alpha \beta}=T_{\alpha \beta}$ the expressions given in the proposition follow.

\section{Main theorem}

We are now in the position of stating and proving our main theorem.

Theorem 1. Let $\left(V^{F L}, g^{F L}\right)$ be a FLRW spacetime and $\left(V^{s t}, g^{s t}\right)$ be a static spacetime, as defined at the beginning of section 3. Let $\left(V^{-}, g^{-}\right)$be an open submanifold of $\left(V^{F L}, g^{F L}\right)$ with $C^{3}$ connected boundary $\Omega^{-}$and $\left(V^{+}, g^{+}\right)$an open submanifold of $\left(V^{\text {st }}, g^{\text {st }}\right)$ with $C^{3}$ connected boundary $\Omega^{+}$. Assume that $\Omega^{-}$is generic (according to definition 3 ).

Then, a $C^{0}$ spacetime $(V, g)$ can be constructed by gluing $V^{+}$and $V^{-}$across their boundaries if and only if the following conditions are satisfied.

(1) In the coordinate system in which the FLRW metric takes the form (31), the boundary $\Omega^{-}$of $V^{-}$is defined by the embedding $\{t, \theta, \phi\} \rightarrow\{t, r=r(t), \theta, \phi\}$, where $r(t)$ is a non-negative $C^{3}$ function. The submanifold $V^{-}$is defined by $\left\{\epsilon_{1} r \geqslant \epsilon_{1} r(t)\right\}$, where $\epsilon_{1}= \pm 1$.

(2) There exists a coordinate system $\{T, t, \theta, \phi\}$ in an open neighbourhood $\mathcal{U}$ of $\Omega^{+}$in $V^{-}$ such that the static metric takes the form

$$
\begin{aligned}
\mathrm{d} s^{2}=-\frac{(\cosh \beta+\mu \sinh \beta)^{2}}{\Delta^{2}} \mathrm{~d} T^{2}+(\mu \cosh \beta+\sinh \beta)^{2} \mathrm{~d} t^{2} \\
+a^{2}(t) \Sigma^{2}(r(t), \epsilon)\left[\left(\mathrm{d} \theta-\left.f(t) \frac{\Sigma_{r}}{\Sigma}\right|_{r=r(t)} \sin \theta \mathrm{d} t\right)^{2}+\sin ^{2} \theta \mathrm{d} \phi^{2}\right],
\end{aligned}
$$

where $\Sigma(r)$ is defined in (24), $\mu \equiv \epsilon_{1} a(\dot{r}+f(t) \cos \theta), \beta(t)$ is given by (27) and $f(t)$, $\Delta(t)$ solve the ODE system (32).

(3) The boundary $\Omega^{+}$is defined in the $\{T, t, \theta, \phi\}$ coordinate system by the embedding $\{t, \theta, \phi\} \rightarrow\{T=T(t), t, \theta, \phi\}$, where $T(t)$ satisfies $\dot{T}(t)=\Delta(t)$.

Remark. If the boundaries $\Omega^{ \pm}$are allowed to have several connected components, then the conditions of the theorem must apply to each one of these connected components. In particular, it follows that two static regions cannot merge and still remain static. Note that this must also hold for two Einstein-Straus vacuoles.

Proof. The necessary part of the theorem has already been proven, except for the explicit form of the metric in the static region. Let us consider the open neighbourhood $\mathcal{U}=I_{1} \times \Pi(\Omega)$ introduced at the beginning of section $4 . \Omega$ and hence $\Pi(\Omega)$ are foliated by 2 -spheres $\left\{S_{t}^{-}\right\}$. Let $q$ be a point on $\mathcal{U}$. Since $\Omega$ and $\Pi(\Omega)$ are diffeomorphic, there is a unique $p \in \Omega$ such that 
$\Pi(q)=\Pi(p)$. Furthermore, there exists a unique value $t_{p}$ such that $p \in S_{t_{p}}^{-}$. Let $\theta_{p}, \phi_{p}$ be the spherical coordinates of $p$ as seen from the FLRW coordinate system (31). Assign to the point $q$ the values $T_{q}$ (static time), $t_{p}, \theta_{p}, \phi_{p}$. It is easy to see that $\{T, t, \theta, \phi\}$ thus constructed is a well defined coordinate system in $\mathcal{U}$. In this coordinate system the static metric takes the form

$\mathrm{d} s^{2}=-D^{2} \mathrm{~d} T^{2}+C^{2} \mathrm{~d} t^{2}+a^{2}(t) \Sigma^{2}(r(t), \epsilon)\left[(\mathrm{d} \theta+H \mathrm{~d} t)^{2}+\sin ^{2} \theta(\mathrm{d} \phi+V \mathrm{~d} t)^{2}\right]$,

where $D, C, H$ and $V$ are unknown functions of $(t, \theta, \phi)$ and we used the fact that the surfaces $T=$ constant, $t=$ constant are 2-spheres of radius $a(t) \Sigma(r(t), \epsilon)$. By construction, the matching hypersurface $\Omega^{+}$is defined by $T=T(t)$ where $T(t)$ is a $C^{3}$ function. Imposing the equality of the first fundamental on $\Omega$ evaluated from the static side and from the FLRW side (see (31)), we find

$$
\begin{aligned}
& C^{2}-D^{2} \dot{T}^{2}=-1+a^{2}(t)(\dot{r}+f(t) \cos (\theta))^{2}, \\
& H=-\left.f \sin (\theta) \frac{\Sigma_{, r}}{\Sigma}\right|_{r=r(t)}, \\
& V=0 .
\end{aligned}
$$

In order to determine $C$ we use (13) and (16) and lemma 3 to obtain

$$
\left.g(\vec{m}, \vec{\xi})\right|_{\Omega}=-D^{2} \Delta
$$

Since $\vec{m}$ is tangent to $\Omega$, orthogonal to the foliation by 2 -spheres and satisfying $g^{F L}(\vec{u}, \vec{m})=-1$ (or, equivalently, $\vec{m}(t)=1$ ), it follows that $\vec{m}=\dot{T} \partial_{T}+\partial_{t}-H \partial_{\theta}$ in the coordinate system $\{T, t, \theta, \phi\}$ for the static region $\mathcal{U}$. Evaluating the scalar product of $\vec{m}$ with $\vec{\xi}=\partial_{T}$ and using (40) we conclude $\dot{T}=\Delta$. The expression for $C$ now follows from (39), the result being $C^{2}=(\mu \cosh \beta+\sinh \beta)^{2}$. This shows that the static metric on $\mathcal{U}$ takes the form (37).

In order to prove the sufficient part of the theorem we only need to check that the matching conditions (1) and (3) are satisfied by the metrics and the hypersurfaces in the theorem. The riggings can be taken to be $\vec{l}_{+}=\partial_{T}$ in the static region and

$\vec{l}_{-}=\frac{\cosh \beta+\mu \sinh \beta}{\Delta}\left[\cosh \beta\left(\partial_{t}-f \cos \theta \partial_{r}+\left.f \frac{\Sigma_{, r}}{\Sigma}\right|_{r=r(t)} \sin \theta \partial_{\theta}\right)-\frac{\sinh \beta}{a} \partial_{r}\right]$

in the FLRW region (in the coordinates where the metric takes the form (31)). The result follows after a straightforward, if somewhat long, calculation.

The ES model is obviously contained in theorem 1. It corresponds to the case in which $f(t)=0$ (so that the static region and its boundary are spherically symmetric) and $\dot{r}=0$, $p=0$ (so that the static region is a vacuum). Furthermore, for most reasonable energymomentum tensors in the static region, proposition 2 forces the static metric and its boundary to be spherically symmetric (see [12] for a detailed discussion). Thus, this theorem implies, in particular, that spherical symmetry is a crucial ingredient in the Einstein-Straus model. Hence, this model is not robust and cannot give a satisfactory answer to the problem of the influence of the cosmic expansion in local physics.

\section{Acknowledgments}

I would like to thank J M M Senovilla, R Vera and B G Schmidt for useful comments on a previous version of this paper. Discussions with Professor W B Bonnor and Professor J Ehlers on the problem of the influence of the cosmic expansion on local physics are gratefully acknowledged. I would also like to thank the Albert Einstein Institute for kind hospitality. 


\section{Appendix}

Let $(\mathcal{V}, \tilde{g})$ be a warped product $\mathcal{V}=\mathbb{R} \times \mathcal{K}$ such that $(\mathcal{K}, g)$ has an isometry group $G$ of positive dimension. The warped product metric is $\tilde{g}=-\mathrm{d} t^{2}+a^{2}(t) g$, where $a(t)$ is a positive function. Let us denote by $\varphi: G \rightarrow \operatorname{Diff}(\mathcal{K})$ the realization of $G$ as a diffeomorphism group of $\mathcal{K}$ and write $\varphi_{h}=\varphi(h), \forall h \in G$. Let $\gamma: \mathbb{R} \rightarrow G$ be a smooth path satisfying $\gamma(0)=e$, where $e$ is the identity of $G$. Fix a point $p \in \mathcal{K}$ and take a sufficiently small open neighbourhood $\mathcal{U}_{p} \subset \mathcal{K}$ of $p$ and a coordinate system $x: \mathcal{U}_{p} \rightarrow \mathbb{R}^{m}$. Choose an open interval $0 \in I_{0} \subset \mathbb{R}$ and an open neighbourhood $\mathcal{W}_{p} \subset \mathcal{U}_{p}$ satisfying $\varphi_{\gamma(t)}^{-1}\left(\mathcal{W}_{p}\right) \subset \mathcal{U}_{p}, \forall t \in I_{0}$. It follows that the map $x_{t}: \mathcal{W}_{p} \rightarrow \mathbb{R}^{m}$ defined by $x_{t}=x \circ \varphi_{\gamma(t)}^{-1}$ is a coordinate system in $\mathcal{W}_{p}$. Obviously, $\left(t, x_{t}\right)$ is a coordinate system on $I_{0} \times \mathcal{W}_{p} \subset \mathcal{V}$. Our aim is to find the explicit form of the metric $\tilde{g}$ in the coordinate system $\left(t, x_{t}\right)$. We first determine the coordinate transformation. For $t \in I_{0}$, define the function

$$
\begin{aligned}
& x \circ \varphi_{\gamma(t)} \circ x^{-1}: x\left(\varphi_{\gamma(t)}^{-1}\left(\mathcal{W}_{p}\right)\right) \subset \mathbb{R}^{m} \rightarrow x\left(\mathcal{W}_{p}\right) \subset \mathbb{R}^{m} \\
& y^{\beta} \rightarrow \Phi^{\alpha}(t, y) \equiv\left(x \circ \varphi_{\gamma(t)} \circ x^{-1}(y)\right)^{\alpha},
\end{aligned}
$$

which clearly satisfies $\Phi^{\alpha}(0, y)=y^{\alpha}$. The functions $\Phi^{\alpha}(t, y)$ define the coordinate transformation from the coordinates $\left(t, x_{t}\right)$ into the coordinates $(t, x)$. Let the line-element in $\left(t, x^{\alpha}\right)$ coordinates be written as

$$
\mathrm{d} s^{2}=-\mathrm{d} t^{2}+a^{2}(t) g_{\alpha \beta}\left(x^{\mu}\right) \mathrm{d} x^{\alpha} \mathrm{d} x^{\beta} .
$$

Since $\varphi_{t}$ is an isometry of $\mathcal{K}$ the following identity holds:

$$
g_{\mu \nu}(y)=g_{\alpha \beta}(\Phi(t, y)) \frac{\partial \Phi^{\alpha}(t, y)}{\partial y^{\mu}} \frac{\partial \Phi^{\beta}(t, y)}{\partial y^{\nu}} .
$$

Using this expression, the metric in the coordinates $\left(t, x_{t}\right)$ becomes easily

$$
\begin{aligned}
\mathrm{d} s^{2}=-\mathrm{d} t^{2}+ & a^{2}(t)\left[g_{\mu \nu}(y) \mathrm{d} y^{\mu} \mathrm{d} y^{\nu}+2 g_{\alpha \beta}(\Phi(t, y)) \frac{\partial \Phi^{\alpha}(t, y)}{\partial y^{\mu}} \frac{\partial \Phi^{\beta}(t, y)}{\partial t} \mathrm{~d} y^{\mu} \mathrm{d} t\right. \\
& \left.+g_{\alpha \beta}(\Phi(t, y)) \frac{\partial \Phi^{\alpha}(t, y)}{\partial t} \frac{\partial \Phi^{\beta}(t, y)}{\partial t} \mathrm{~d} t^{2}\right],
\end{aligned}
$$

where $g_{\alpha \beta}$ are the same functions as in (A1). In order to elaborate this expression further, we need to find $\partial_{t} \Phi^{\alpha}(t, y)$. Let us define $U_{t}(\lambda) \equiv \gamma(t+\lambda) \gamma^{-1}(t)$, which is, for each $t$, a smooth curve in $G$ satisfying $U_{t}(0)=e$. Its tangent vector at the identity,

$$
U^{\prime}(t)=\left.\frac{\mathrm{d} U_{t}(\lambda)}{\mathrm{d} \lambda}\right|_{\lambda=0},
$$

is (for each $t$ ) an element of the Lie algebra $\mathfrak{g}$ of $G$. Using the differential of $\varphi: G \rightarrow \operatorname{Diff}(\mathcal{K})$, we can associate to $U^{\prime}(t)$ a Killing vector field $\vec{v}_{t}$ on $\mathcal{K}$. From the definition of Killing vector field we have

$$
\left.\frac{\mathrm{d}\left(x \circ \phi_{U_{t}(\lambda)} \circ x^{-1}(y)\right)^{\alpha}}{\mathrm{d} \lambda}\right|_{\lambda=0}=v_{t}^{\alpha}(y),
$$

where, by definition, $v_{t}^{\alpha}(y)$ are the components of $\vec{v}_{t}$ in the coordinate system $x$ evaluated at the coordinate values $y$. Using the group properties, it is straightforward to show that

$$
\frac{\partial \Phi^{\alpha}(t, y)}{\partial t}=v_{t}^{\alpha}(\Phi(t, y))
$$


Let us now define the function $\Psi^{\alpha}(t, z)$ as the inverse of $\Phi(t, y)^{\alpha}$ with respect to the variables $y^{\alpha}$. Inserting

$$
\delta_{\mu}^{\alpha}=\left.\frac{\partial \Psi^{\alpha}(t, z)}{\partial z^{\beta}}\right|_{z=\Phi(t, y)} \frac{\partial \Phi^{\beta}(t, y)}{\partial y^{\mu}},
$$

into (A3) and using (A4) we find, after taking into account (A2)

$$
\begin{aligned}
\mathrm{d} s^{2}=-\mathrm{d} t^{2}+ & a^{2}(t) g_{\mu \nu}(y)\left(\mathrm{d} y^{\mu}+\left.v_{t}^{\sigma}(\Phi(t, y)) \frac{\partial \Psi^{\mu}(t, z)}{\partial z^{\sigma}}\right|_{z=\Phi(t, y)} \mathrm{d} t\right) \\
& \times\left(\mathrm{d} y^{\nu}+\left.v_{t}^{\rho}(\Phi(t, y)) \frac{\partial \Psi^{\nu}(t, z)}{\partial z^{\rho}}\right|_{z=\Phi(t, y)} \mathrm{d} t\right),
\end{aligned}
$$

which can be written more transparently by using the definition of a differential map,

$\mathrm{d} s^{2}=-\mathrm{d} t^{2}+a^{2}(t) g_{\mu \nu}(y)\left(\mathrm{d} y^{\mu}+\left[\Phi_{\gamma(t) \star}^{-1}\left(\vec{v}_{t}\right)\right]^{\mu}(y) \mathrm{d} t\right)\left(\mathrm{d} y^{\nu}+\left[\Phi_{\gamma(t) \star}^{-1}\left(\vec{v}_{t}\right)\right]^{\nu}(y) \mathrm{d} t\right)$,

where the $\star$ denotes a differential map and where $\left[\Phi_{\gamma(t) \star}^{-1}\left(\vec{v}_{t}\right)\right]^{\nu}(y)$ are the components of the vector in brackets in the coordinate system $x$ and evaluated at the coordinate values $y$. The differential map of an isometry is well known to map Killing vector fields into Killing vector fields. Thus $\vec{\eta}_{t} \equiv \Phi_{\gamma(t) \star}^{-1}\left(\vec{v}_{t}\right)$ is a Killing vector field of $\mathcal{K}$ for each $t$. Summarizing, the metric can be written as

$$
\mathrm{d} s^{2}=-\mathrm{d} t^{2}+a^{2}(t) g_{\mu \nu}(y)\left(\mathrm{d} y^{\mu}+\eta_{t}^{\mu}(y) \mathrm{d} t\right)\left(\mathrm{d} y^{\nu}+\eta_{t}^{\nu}(y) \mathrm{d} t\right),
$$

where $g_{\mu \nu}(y)$ are the metric components of $g$ in the coordinate system $x$, evaluated at the values $y$ and, for each $t \in I_{0} \subset \mathbb{R}, \eta_{t}^{v}(y)$ are the components of a Killing vector of $\mathcal{K}$ written in the original coordinates $x$ and taken at the coordinate value $y$.

The case considered in the main text corresponds to $\mathcal{K}=\mathcal{M}$, i.e. a space of constant curvature. In section 3 we defined a curve $\sigma: I_{2} \rightarrow \mathcal{M}$ which described the spatial projection of the motion of the centre of the 2-spheres foliating the matching hypersurface $\Omega^{-}$and we fixed a point $p=\sigma\left(t_{0}\right)$. Consider the tangent vector $\dot{\sigma}\left(t_{0}\right)$ and choose a spherically symmetric coordinate $\{r, \theta, \phi\}$ system in $\mathcal{M}$ centred at $p$ such that $\dot{\sigma}\left(t_{0}\right)$ is parallel to the axis $\theta=0$ (this is well defined even when $\dot{\sigma}\left(t_{0}\right)=\overrightarrow{0}$ ). Taking $x$ to be this spherical coordinate system, the coordinates defined in section 3 adapted to the matching hypersurface correspond to the coordinates $\left(t, x_{t}\right)$ considered in this appendix. Furthermore, the path $\gamma(t)$ was chosen such that the tangent vector $U^{\prime}(t)$ has only components in the transvection vectors of $\varphi_{\gamma(t)}(p)$. This implies, from the properties of symmetric spaces, that $\vec{\eta}_{t}$ defined above is a transvection vector at $p$. A basis of transvection vectors at $p$ is the coordinate system $x$ is given by (26). Hence $\vec{\eta}_{t}=h^{i}(t) \vec{\eta}_{i}$ follows readily. Finally, $h^{2}\left(t_{0}\right)=0$ and $h^{3}\left(t_{0}\right)=0$ is a consequence of having adapted the spherical coordinate system $x$ to the direction of $\dot{\sigma}\left(t_{0}\right)$. Under these conditions (25) is obtained directly from (A5).

\section{References}

[1] McVittie G C 1933 The mass-particle in an expanding universe Mon. Not. R. Astron. Soc. 93325

[2] Järnefeld G 1940 Zur relativistischen Perihelbewegung der Planetenbahnen Ann. Acad. Soc. Sci. Fennicae A 553

[3] Sussman R 1988 On spherical symmetry shear-free perfect fluid configurations (neutral and charged) III. Global view J. Math. Phys. 29 1177-211

[4] Nolan B C 1998 A point mass in an isotropic universe: existence, uniqueness and basic properties Phys. Rev. D 58064006 1-10

Nolan B C 1999 A point mass in an isotropic universe: II. Global properties Class. Quantum Grav. 16 1227-54 
Nolan B C 1999 A point mass in an isotropic universe: III. The region $R \leqslant 2 m$ Class. Quantum Grav. 16 3183-91

[5] Einstein A and Straus E G 1945 The influence of the expansion of space on the gravitation fields surrounding the individual stars Rev. Mod. Phys. 17 120-4

Einstein A and Straus E G 1946 Corrections and additional remarks to our paper: The influence of the expansion of space on the gravitation fields surrounding the individual stars Rev. Mod. Phys. 18 148-9 (erratum)

[6] Bonnor W B 1996 The cosmic expansion and local dynamics Mon. Not. R. Astron. Soc. 282 1467-9

[7] Krasiński A 1997 Inhomogeneous Cosmological Models (Cambridge: Cambridge University Press)

[8] Bonnor W B 1999 Size of a hydrogen atom in the expanding universe Class. Quantum Grav. 16 1313-21

[9] Mars M, Senovilla J M M and Vera R 1999 Atoms against the Universe Phys. World 7 20-1

[10] Bonnor W B 2000 A generalization of the Einstein-Straus vacuole Class. Quantum Grav. 17 2739-48

[11] Senovilla J M M and Vera R 1997 Impossibility of the cylindrically symmetric Einstein-Straus model Phys. Rev. Lett. 78 2284-7

[12] Mars M 1998 Axially symmetric Einstein-Straus model Phys. Rev. D 57 3389-400

[13] Kramer D, Stephani H, MacCallum M A H and Herlt E 1980 Exact Solutions of Einstein's Field Equations (Cambridge: Cambridge University Press)

[14] Schücking E 1954 Das Schwarzschildsche Linienelement und die Expansion des Weltalls Z. Phys. 137 595-603

[15] Ellis G F R 1984 Relativistic cosmology: its nature, aims and problems General Relativity and Gravitation ed B Bertotti, F de Felici and A Pascolini (Dordrecht: Reidel) pp 215-88

Krasiński A 1997 Inhomogeneous Cosmological Models (Cambridge: Cambridge University Press) ch VIII

Mars M and Zalaletdinov R 1997 Space-time averages in macroscopic gravity and volume preserving coordinates J. Math. Phys. 38 4741-57

[16] Darmois G 1927 Mémorial des Sciences Mathématiques (Paris: Gauthier-Villars) Fascicule 25

[17] Mars M and Senovilla J M M 1993 Geometry of general hypersurfaces in spacetime: junction conditions Class. Quantum Grav. 10 1865-97

[18] Hirsch M W 1976 Differential Topology (Graduate Texts in Mathematics vol 33) (New York: Springer)

[19] Kobayashi S and Nomizu K 1963 Foundations of Differential Geometry vol II (New York: Interscience)

[20] O’Neill B 1997 Elementary Differential Geometry (New York: Academic) p 419

[21] Berger M and Gostiaux G 1988 Differential Geometry: Manifolds, Curves and Surfaces (Graduate Texts in Mathematics vol 115) (New York: Springer)

[22] Spivak M 1975 A Comprehensive Introduction to Differential Geometry vol IV (Berkeley, CA: Publish or Perish)

[23] Jost J 1995 Riemannian Geometry and Geometric Analysis (Universitext) (Berlin: Springer)

[24] Alcubierre M 1994 The warp drive: hyper-fast travel within general relativity Class. Quantum Grav. 11 L73-7 